\title{
Association of Type of Splint, Rationale for Splinting and Survival of Splint, Based on Different Clinical Scenarios with Long Term Follow Up in Chennai Population
}

\author{
Siddharth Narayan ${ }^{1}$, Sankari Malaiappan ${ }^{2}$ \\ 1, 2 Department of Periodontology, Saveetha Dental College, Chennai, Tamil Nadu, India.
}

\section{ABSTRACT}

\section{BACKGROUND}

The revival of older treatment modalities like splinting requires the assessment of newer patient centric parameters like survival, compliance, anaesthetics apart from changing the biomaterials employed alone. The purpose of this study was to assess the association between type of splint, chief complaint and survival of splint based on different clinical scenarios with long term follow-up.

\section{METHODS}

The present study was a prospective university based clinical trial with convenience sampling to include clinical scenarios which required splinting in a Chennai Dental College. Ninety-five adult patients were included in the study comprising 50 men and 45 women, aged between 19 - 65 years where parameters like demographic distribution, type of splint, location of the splint, patient compliance and survival were included. The primary end point of the present study was recall of patients periodically every month till 6 - 8 months to assess the duration of survival of splints and associate the same with age, gender, patient compliance, type of splint and rationale for splinting.

\section{RESULTS}

Among the 95 patients included (524 teeth splinted together), all patients completed the treatment protocol, stainless steel wire composite splints were given to 70 patients while composite mesh splints were used in 20 patients. A likelihood ratio of $0.024(\mathrm{P}<0.05)$ was seen while associating composite mesh splints, rationale for use, survival time and patient compliance.

\section{CONCLUSIONS}

Within the limits of the present study, a conclusion was made that one must emphasise on the importance of retaining natural teeth based on appropriate clinical scenarios where maximum benefit could be obtained among compliant patients.

\section{KEY WORDS}

Periodontal Splints, Survival Rate, Patient Compliance, Rationale for Splinting, Tooth Fractures, Tooth Reimplantation
Corresponding Author: Dr. Siddharth Narayan, Saveetha Dental College, No 162, Poonamallee High Road, Chennai, Tamil Nadu, India. E-mail: dr.siddharthnarayan@gmail.com

DOI: $10.14260 / \mathrm{jemds} / 2021 / 462$

How to Cite This Article:

Narayan S, Malaiappan S. Association of type of splint, rationale for splinting and survival of splint, based on different clinical scenarios with long term follow up in Chennai population. J Evolution Med Dent Sci 2021;10(30):2260-2265, DOI: $10.14260 / \mathrm{jemds} / 2021 / 462$

Submission 05-05-2021, Peer Review 05-07-2021, Acceptance 12-07-2021, Published 26-07-2021.

Copyright (c) 2021 Siddharth Narayan et al. This is an open access article distributed under Creative Commons Attribution License [Attribution 4.0 International (CC BY 4.0)] 


\section{BACKGROUND}

Periodontology is a combined science dealing with both hard as well as soft tissue which takes an interdisciplinary dental approach to treat chief complaints while simultaneously keeping in mind any other problems which may arise in the future. Considering periodontitis as an established multifactorial tissue invasive infection of prime bacterial origin, inadvertent repetitive mechanical debridement alone is not sufficient in treatment and requires antimicrobial therapy as well. ${ }^{1}$ The most common complaints with which patients are referred to this department is tooth mobility along with bleeding gums and difficulty in chewing.

Early animal study models have suggested progressive attachment loss around involved teeth which eventually resulted in the increased severity of mobility as compared to teeth which were splinted.2,3 Splinting multiple teeth together causes dissipation of forces acting on individual teeth to a group of teeth enabling them to act as a functional unit passively, without actual application of any force on adjacent teeth. Though there is limited histological or scientific proof for such a rationale, the deleterious effect of traumatic occlusion on the periodontium have been extensively documented and studied over year. ${ }^{4}$ Many procedures centred at the rehabilitation of periodontium and level of bone loss start by addressing trauma from occlusion in the form of coronoplasty, orthodontic treatment at phase I therapy itself. They are aimed to temporarily relieve and reduce the load acting on the periodontium of affected teeth which is then distributed on adjacent teeth especially in terminal stages of periodontitis. Apart from just patients, splinting also installs some extent of confidence within practitioners to carry on with surgical intervention on patients without the fear of aggravated post-surgical mobility or exfoliation during flap surgery. ${ }^{5}$ Chief complaint related medicine is already a part of current day dentistry where the chief complaint of the patient is seen as the prime objective behind therapy. The future however is all about personalized periodontal therapy which is tailored to a particular medical reality of a specific patient. ${ }^{6}$ This future also sees the return of treatment modalities like splinting as patients have come to understand and appreciate the value of teeth as compared to prosthesis which only act as the next best thing and not a substitute. The revival of such an age-old treatment modality requires newer biomaterials enhancing treatment outcomes without changing crucial concepts. This can be better understood with the sudden active surge of tooth-adhesion biomaterials and composites instead of conventional stainless-steel wires with good material strength but no chemical bonding to tooth structure. ${ }^{7}$

The purpose of the present study was to assess the association between type of splint, chief complaint and survival of splint based on different clinical scenarios with long term follow-up.

\section{METHODS}

The present study was undertaken in a university-based setup as a prospective clinical trial with convenience sampling due to selective inclusion of cases based on the need for splinting for various clinical scenarios by the Department of Periodontology in a Chennai (India) based Dental College. The study protocol was approved by the University Ethical Committee and research board with Approval No. IHEC / SDC / PERIO - 1802 / 20 / 28 and written consent was obtained from all included participants for the use of their demographic data and pictures for the purpose of research. All patients included in the present study were individuals who present to the Chennai based dental college and hospital from $1^{\text {st }}$ August 2020 to $1^{\text {st }}$ April 2021 with a chief compliant which required either stainless steel or composite mesh wire splints to stabilise teeth. There is lack of evidence in literature of clinical trials using splint in assessing periodontal parameters, the sample size for present study was based on study performed by Akcali A et al. which used a sample of 14 patients assessing survival for 6 months. ${ }^{8}$ Ninety-five adult patients were included in the study comprising 50 men and 45 women, aged between $19-65$ years. (Table 1) Only permanent maxillary and mandibular teeth in adult patients were included in the study where clinical assessment suggested the need for splinting (SN) under the guidance of (SM).

Criteria for patient exclusion was (1) Patients below the age of 18 and above the age of 70 (2) Prior dental appointments for fillings in the tooth requiring splinting (3) Grade 3 tooth mobility (4) Severe malocclusion which would affect force dissipation while splinting (5) Radiographic evidence of large radiolucency or complete absence of supporting bone. The primary end point of the present study was recall of patients periodically every month till $6-8$ months to assess the duration of survival of splints and associate the same with patient compliance, type of splint and rationale for usage. Prior to any form of intervention, a detailed case sheet was recorded illustrating age, gender, demographic distribution, chief complaint along with clinical features like the location of the splint, type of splint utilised, and teeth involved. All included patients were subjected to full-mouth scaling with an ultrasonic scaler (Suprasson P5 Booster; Satelec Scalers) to remove calculus deposits and were instructed on how to maintain oral hygiene.

After 1 week, patients received the type of splint based on their aesthetic comfort or severity of progression of mobility by a single operator to standardise the level of care and clinical skill provided. (Figure 2). Additional parameters were analysed such as patient compliance where individuals who reported for periodic follow up on all appointments every one month without any complaints over a period of 6 months were recorded as compliant patients while individuals who missed all follow up appointments every month and returned only for modifying the splints were recorded as noncompliant patients. Rationale for splinting of chief complaint was recorded under the following categories namely trauma, provisional splint (under active periodontal therapy), permanent splint (under periodontal maintenance phase after prior treatment), tooth fracture and reimplantation.(Figure 3) All data collected in the present study was obtained and analysed by a single operator (SN) under the direct supervision of the second author. 

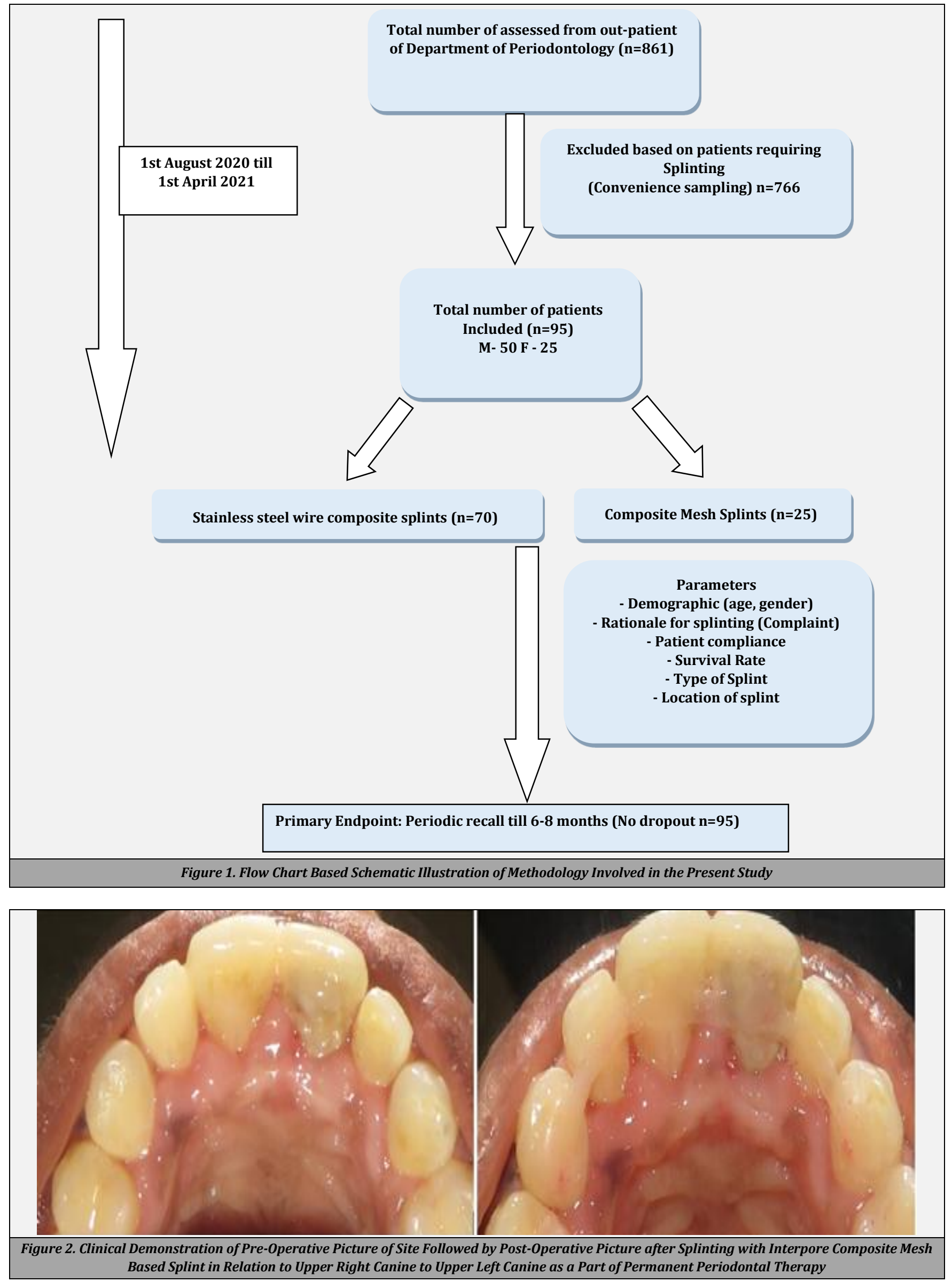

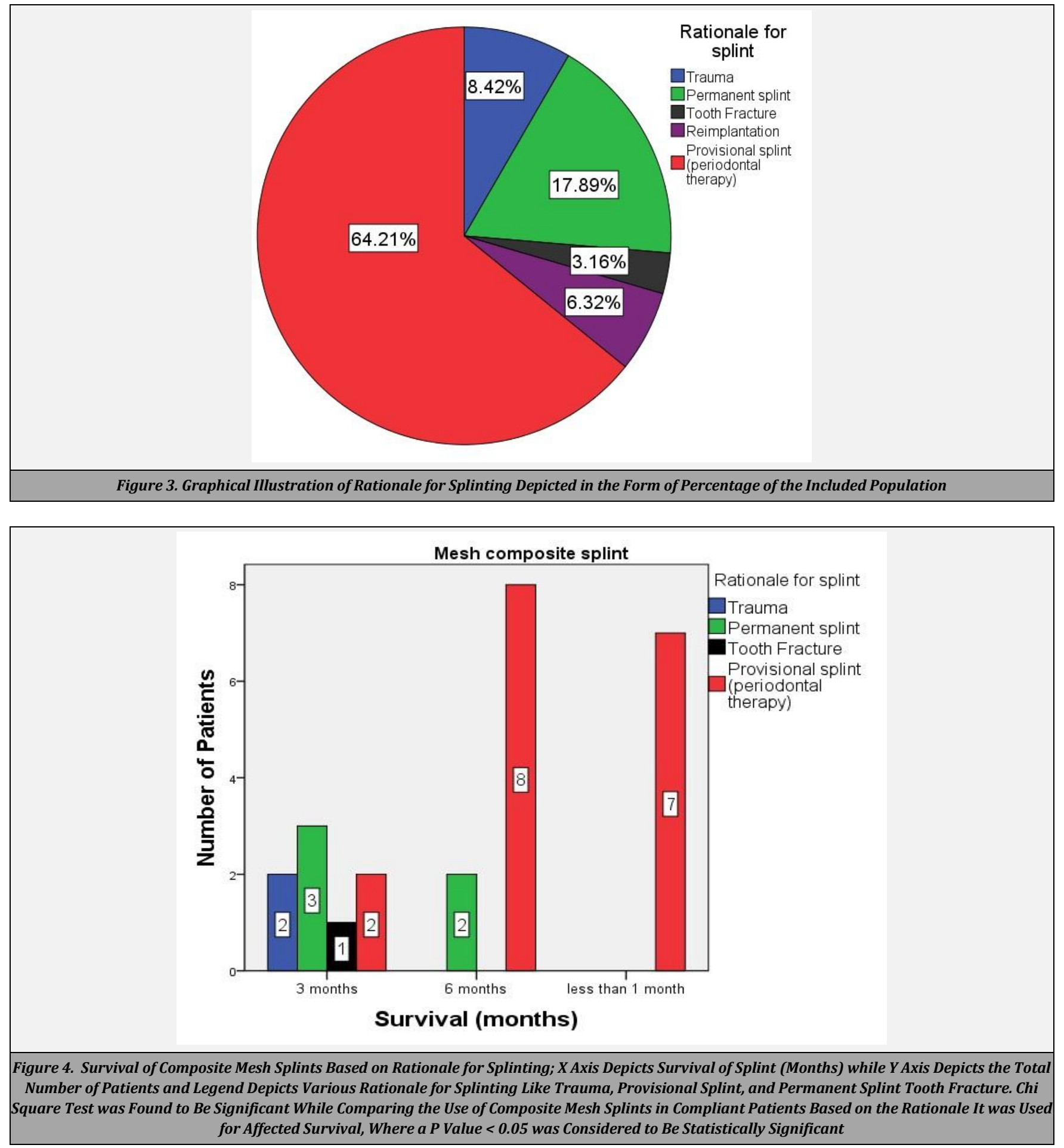

\section{Statistical Analysis}

All data obtained from the present study were expressed in the form of mean \pm standard deviation and assessed for statistical significance using statistical package for social sciences (SPSS) version 23, where a statistical significance of $\mathrm{P}<0.05$ was considered statistically significant. Independent variable prior to initiation of the study were: age, gender, location of splint and rationale for splinting which were confirmed using chi square $(\mathrm{P}>0.05)$ to rule out selection bias. However, the likelihood ratio using chi square was found to be 0.024 while comparing the association between composite mesh splints, patient compliance and survival of splints was statistically significant. $(\mathrm{P}<0.05)$

\section{RESULTS}

Among the 95 patients included (524 teeth splinted together), all patients completed the treatment protocol, and no patients were lost at any stage of the study due to patient drop out irrelevant of compliance. Stainless steel wire composite splints were given to seventy patients while composite mesh splints were used in twenty-five patients. Splint survival was assessed once every month for a follow up period of 6 months which revealed $54.7 \%$ of the splints survived for 6 , while $36.8 \%$ of the splints survived for 3 months and $8.4 \%$ survived for less than a month without 
modifications. Splints were used in anterior teeth including both upper and lower in about 80 patients while 15 patient received splints for posterior teeth which were most commonly stainless-steel wire splints stabilised with composite resin.

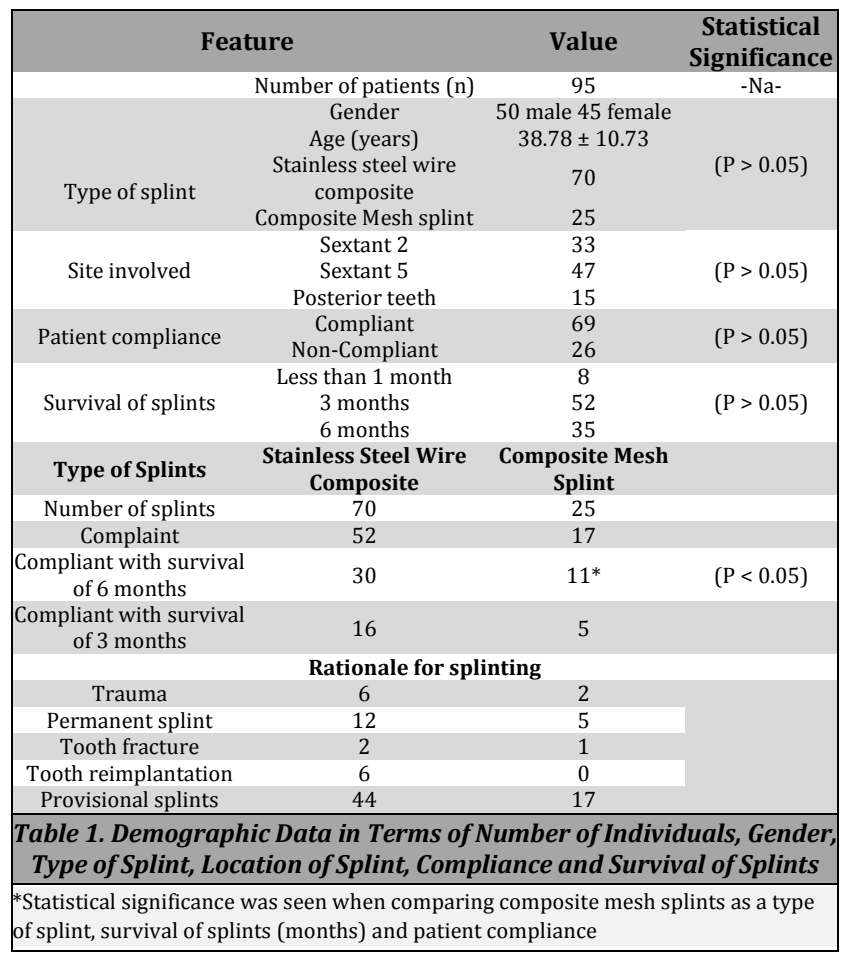

\section{DISCUSSION}

Periodontal prognosis of a tooth is one of the most common reasons for referral to a periodontist from other dental specialities for long term prosthetic rehabilitation. The assessment of prognosis is complicated and often requires a comprehensive medical history, ruling out of systemic diseases while considering all local factors affecting treatment modalities at the site. The local factors affecting treatment modalities include furcation involvement, mobility, root morphology, vitality, endodontic prognosis, and restorability of tooth back into function. Among all these factors, mobility is a relative term and despite a universally followed system to classify it such as S.C Millers grading of tooth mobility (1950), it often is subjective when it comes to grade 1 and grade 2 mobility while there is clarity in dual planes of mobility to allocate grade 3 mobility. Mobility may not be considered as a major clinical determinant of prognosis but apart from just its clinical significance, it also acts as a constant reminder to the patient affecting his overall quality of life. ${ }^{9}$ Simultaneously, untreated progressive periodontitis would lead to functional, aesthetic difficulties as well as discomfort which consequently reduced oral healthrelated quality of life and tooth loss. ${ }^{10}$ Natural teeth are truly one of a kind which can rarely ever be replaced, a common misconception is the prosthetic rehabilitation of extracted missing teeth as an alternative though it actually is the only next best thing as compared to teeth. Apart from function, aesthetic and smile related confidence, teeth firmly attached to periodontal ligament induce a sense of proprioception, the awareness of the position and movement of a body in 3-D environment without the actual visualization of the same. This feature of teeth allows one feel, sense and perceives food while biting into them for example the perception of biting into rice without actually seeing rice going into the mouth.

Splinting does not completely eliminate occlusal stress as functional stimuli exerted in the long axis of the teeth is still essential for dynamic bone level turnover preventing disuse atrophy of teeth. The function of a splint is to distribute and redirect functional and parafunctional forces to bring them within tolerance of the supporting tissue and to reduce mobility. It was also observed that stainless steel wire composite splints were the preferred treatment modality in cases where reimplantation or tooth fracture was seen which could be attributed to the desirable properties of stainless steel in terms of compressive and tensile strength.

Patient compliance and smoking was analysed and associated with patient's overall oral health perception suggesting a positive association between both in some studies. ${ }^{11}$ As oral health ultimately affects periodontal outcome and maintenance therapy, the authors considered it as a possible psychological factor which could affect the survival rate of splints. The present study showed no statistical significance between age, gender, patient compliance and survival of splints. However, evidence in recent studies suggested that older individuals showed better oral hygiene related quality of life and the authors suggested this to be indicative of some kind of coping mechanism among the elderly. ${ }^{12}$

The present study showed a likelihood ratio of 0.024 where composite mesh splints would survive for a greater time period based on the rationale it was used for and patient compliance. These findings were seen independent of any direct statistically significant relation between compliance or rationale for splinting and survival of the splint. Studies in literature have found a greater reduction in mobility from grade II mobility to grade I mobility while using stainless steel wire composite resin splints among age groups of 20 40 years with a lifespan of 6 months $24 \%$ of the time but due to their unaesthetic presence they are not always the ideal treatment of choice in aesthetic zones. ${ }^{13}$

Patient compliance has been linked to dental health care maintenance since 1990s where simple techniques such as complete disclosure of information, positive rapport and periodic phone call reminders were found to be an effective means in improving oral hygiene. ${ }^{14}$ The clinical significance of such a small numeric value could always be questioned. However, it has always been considered as some of the factors which affects treatment outcome (rationale and compliance) in the management of long term chronic inflammatory conditions. Long term clinical trials with larger samples are required to confirm the findings of the present study but the present study could pave way for studies relating patient compliance, rationale for therapy, ultimate treatment goals and patient satisfaction.

\section{CONCLUSIONS}

Within the limits of this study, it can be concluded that emphasis must be made not only on the importance of 
retaining the natural value of teeth but also the need to assess appropriate clinical scenarios where maximum benefit can be obtained in compliant patients.

Data sharing statement provided by the authors is available with the full text of this article at jemds.com.

Financial or other competing interests: None.

Disclosure forms provided by the authors are available with the full text of this article at jemds.com.

\section{REFERENCES}

[1] Slots J, Rams TE. Antibiotics in periodontal therapy: advantages and disadvantages. J Clin Periodontol 1990;17(7(Pt 2)):479-93.

[2] Kathariya R, Devanoorkar A, Golani R, et al. To splint or not to splint: the current status of periodontal splinting. J Int Acad Periodontol 2016;18(2):45-56.

[3] Ericsson I, Giargia M, Lindhe J, et al. Progression of periodontal tissue destruction at splinted/non-splinted teeth. An experimental study in the dog. J Clin Periodontol 1993;20(10):693-8.

[4] Fan J, Caton JG. Occlusal trauma and excessive occlusal forces: narrative review, case definitions and diagnostic considerations. J Periodontol 2018;89(Suppl 1):S214-22.

[5] Quirynen M, Mongardini C, Lambrechts P. A long-term evaluation of composite-bonded natural/resin teeth as replacement of lower incisors with terminal periodontitis. J Periodontol 1999;70(2):205-12.

[6] Bartold PM. Lifestyle and periodontitis: the emergence of personalized periodontics. Periodontol 2000 2018;78(1):7-11.
[7] Rauch A, Mehlhorn S, Mühle $M$, et al. A glass fiberreinforced resin composite splint to stabilize and replace teeth in a periodontally compromised patient. Case Rep Dent 2020;2020:8886418.

[8] Akcalı A, Gümüş P, Özcan M. Clinical comparison of fiberreinforced composite and stainless steel wire for splinting periodontally treated mobile teeth. Brazilian Dental Science 2014;17(3):39-49.

[9] Lopes MWF, Gusmao ES, Alves RV, et al. The impact of chronic periodontitis on quality of life in Brazilian subjects. Acta Stomatol Croat 2009;43(2):89-98.

[10] De Freitas Borges T, Regalo SC, Taba M Jr, et al. Changes in masticatory performance and quality of life in individuals with chronic periodontitis. J Periodontol 2013;84(3):325-31.

[11] El Sayed N, Baeumer A, El Sayed S, et al. Twenty years later: oral health-related quality of life and standard of treatment in patients with chronic periodontitis. J Periodontol 2019;90(4):323-30.

[12] Graetz C, Schwalbach M, Seidel M, et al. Oral healthrelated quality of life impacts are low 27 years after periodontal therapy. J Clin Periodontol 2020;47(8):95261.

[13] Narayan S, Lochana GP. The assessment of longevity and efficacy of different types of splints used in periodontal therapy: a single center epidemiological retrospective study. Indian J Forensic Med Toxicol 2020;14(4):613846.

[14] Poplinger A. Adherence to oral hygiene and dental selfcare. Refuat Hapeh Vehashinayim 1993 2010;27(2):3863. 